\title{
On a functional equation involving iterates and powers
}

Janusz Morawiec

\section{"Correspondence:}

morawiec@math.us.edu.pl

Institute of Mathematics, University

of Silesia, Bankowa 14, Katowice,

40-007, Poland

\section{Abstract}

We present a complete list of all continuous solutions $f:(0,+\infty) \rightarrow(0,+\infty)$ of the equation $f^{2}(x)=\gamma[f(x)]^{\alpha} x^{\beta}$, where $\alpha, \beta$ and $\gamma>0$ are given real numbers.

MSC: Primary 39B22; secondary 39B12; 26A18

Keywords: iterative functional equation; continuous solution; bijection

\section{Introduction}

In this note we give a complete list of all continuous solutions $f:(0,+\infty) \rightarrow(0,+\infty)$ of the equation

$$
f^{2}(x)=\gamma[f(x)]^{\alpha} x^{\beta}
$$

where $\alpha, \beta$ and $\gamma>0$ are given real numbers; here and throughout, $f^{2}$ denotes the second iterate of $f$. The motivation for writing this note was two problems concerning continuous solutions $f:(0,+\infty) \rightarrow(0,+\infty)$ of some special cases of equation (1.1) (see [1, Problem 11, p.312] and [2, Problem 5, p.22]) as well as conference reports and papers on both problems (see [3, 4] and [5-8]). Let us mention that the problem from booklet [2] is wrongly solved in this booklet. To see that the problem from [1] concerns really equation (1.1) observe that from Remark 1.1 below it follows that in the case where $\beta \neq 0$ equation (1.1) can be rewritten in the form

$$
f(x)\left[f^{-1}(x)\right]^{-\beta}=\gamma x^{\alpha}
$$

Remark 1.1 Assume $\beta \neq 0$. Then every continuous solution $f:(0,+\infty) \rightarrow(0,+\infty)$ of equation $(1.1)$ is strictly monotone and maps $(0,+\infty)$ onto $(0,+\infty)$.

Proof Fix $x, y \in(0,+\infty)$ and assume that $f(x)=f(y)$. Then by (1.1) we get

$$
\gamma[f(x)]^{\alpha} x^{\beta}=f^{2}(x)=f^{2}(y)=\gamma[f(y)]^{\alpha} y^{\beta}=\gamma[f(x)]^{\alpha} y^{\beta},
$$

and since $\beta \neq 0$, we obtain $x=y$. Thus $f$ is injective. This jointly with continuity implies strict monotonicity.

O2014 Morawiec; licensee Springer. This is an Open Access article distributed under the terms of the Creative Commons Attribution License (http://creativecommons.org/licenses/by/2.0), which permits unrestricted use, distribution, and reproduction in any medium, provided the original work is properly cited. 
Now suppose that, contrary to our claim, $\lim _{x \rightarrow 0} f(x) \in(0,+\infty)$. Then, by the continuity of $f$ and (1.1), we obtain

$$
f\left(\lim _{x \rightarrow 0} f(x)\right)=\lim _{x \rightarrow 0} f^{2}(x)=\lim _{x \rightarrow 0} \gamma[f(x)]^{\alpha} x^{\beta} \in\{0,+\infty\}
$$

a contradiction. Thus $\lim _{x \rightarrow 0} f(x) \in\{0,+\infty\}$. In the same manner we can prove that $\lim _{x \rightarrow+\infty} f(x) \in\{0,+\infty\}$.

\section{Main results}

To give a complete list of all continuous solutions $f:(0,+\infty) \rightarrow(0,+\infty)$ of equation (1.1), we will split our consideration into the following three cases: $\beta=0, \alpha=0 \neq \beta$ and $\alpha \neq$ $0 \neq \beta$. It turns out that the description of all continuous solutions of equation (1.1) in the first case is quite easy; whereas in the third case it is much more complicated than in the second one.

\subsection{The case $\beta=0$}

If $\beta=0$, then equation (1.1) reduces to the equation

$$
f^{2}(x)=\gamma[f(x)]^{\alpha}
$$

Equation (2.1) was examined in [9-11]; $c f$. also [12,13] and the references therein.

We begin with a (rather obvious and simple) characterization of general solutions $f:(0,+\infty) \rightarrow(0,+\infty)$ of equation $(2.1)$

Proposition 2.1 A function $f:(0,+\infty) \rightarrow(0,+\infty)$ satisfies $(2.1)$ if and only if

$$
f(x)=\gamma x^{\alpha}
$$

for all $x \in f((0,+\infty))$.

Proof $(\Rightarrow)$ If a function $f:(0,+\infty) \rightarrow(0,+\infty)$ satisfies $(1.1)$, then for every $x=f(z) \in$ $f((0,+\infty))$ we have $f(x)=f^{2}(z)=\gamma[f(z)]^{\alpha}=\gamma x^{\alpha}$.

$(\Leftarrow)$ Fix a function $f:(0,+\infty) \rightarrow(0,+\infty)$ satisfying $(2.2)$ for all $x \in f((0,+\infty))$. Then, for every $x \in(0,+\infty)$, we have $f(x) \in f((0,+\infty))$. Now, putting $f(x)$ in place of $x$ in $(2.2)$, we obtain (2.1).

From Proposition 2.1 we obtain the following description of all continuous solutions $f:(0,+\infty) \rightarrow(0,+\infty)$ of equation $(2.1)$.

Corollary 2.2 Let $f:(0,+\infty) \rightarrow(0,+\infty)$ be a continuous solution of equation (2.1). Then either $f$ has form $(2.2)$ for all $x \in(0,+\infty)$ or there exists a proper subinterval I (open or closed or closed on one side; possible infinite or degenerated to a single point) of the halfline $(0,+\infty)$ satisfying

$$
\gamma x^{\alpha} \in I \quad \text { for all } x \in I
$$


such that $f$ has form (2.2) for all $x \in I$,

$$
\lim _{x \rightarrow y} f(x)=\gamma y^{\alpha} \quad \text { for all } y \in\{\inf I, \sup I\} \backslash\{0,+\infty\}
$$

and

$$
f((0,+\infty) \backslash I) \subset I
$$

Moreover:

(i) If $\alpha<-1$, then $I=\left\{\gamma^{\frac{1}{1-\alpha}}\right\}$;

(ii) If $\alpha=-1$, then $\operatorname{cl} I=\left[A, \frac{\gamma}{A}\right]$ with arbitrary $A \in(0, \sqrt{\gamma}]$;

(iii) If $\alpha \in(-1,0)$, then $\mathrm{cl} I=[A, B]$ with arbitrary $A \in\left(0, \gamma^{\frac{1}{1-\alpha}}\right]$ and $B \in\left[\gamma^{\frac{1}{1-\alpha}},+\infty\right)$;

(iv) If $\alpha \in[0,1)$, then either $\operatorname{cl} I=[0, B]$ or $\operatorname{cl} I=[A, B]$ or $\operatorname{cl} I=[A,+\infty)$ with arbitrary $A \in\left(0, \gamma^{\frac{1}{1-\alpha}}\right]$ and $B \in\left[\gamma^{\frac{1}{1-\alpha}},+\infty\right)$;

(v) If $\alpha=1<\gamma$, then $\operatorname{cl} I=[A,+\infty)$ with arbitrary $A \in(0,+\infty)$;

(vi) If $\alpha=1=\gamma$, then no restriction on I;

(vii) If $\alpha=1>\gamma$, then $\mathrm{cl} I=(0, B]$ with arbitrary $B \in(0,+\infty)$;

(viii) If $\alpha \in(1,+\infty)$, then either $I=\left\{\gamma^{\frac{1}{1-\alpha}}\right\}$ or $\mathrm{cl} I=[0, B]$ or $\mathrm{cl} I=[A,+\infty)$ with arbitrary $A \in\left[\gamma^{\frac{1}{1-\alpha}},+\infty\right)$ and $B \in\left(0, \gamma^{\frac{1}{1-\alpha}}\right]$.

Proof Put $I=f((0,+\infty))$.

If $I=(0,+\infty)$, then $(2.2)$ holds for all $x \in(0,+\infty)$ by Proposition 2.1. Therefore, to the end of the proof, we assume that $I \neq(0,+\infty)$.

Since $f$ is continuous, it follows that $I$ is an interval. By Proposition 2.1 we see that (2.2) holds for all $x \in I$. Thus (2.3) holds. Condition (2.4) follows from the continuity of $f$ and condition (2.5) is a consequence of the definition of $I$. This completes the proof of the main part of the result.

To prove the moreover part put $A=\inf I$ and $B=\sup I$. Since $I \neq(0,+\infty)$, it follows that $0<A$ or $B<+\infty$.

Assume first that $\alpha<0$.

We will show that $0<A$ and $B<+\infty$.

If $B=+\infty$, then (2.3) implies $0=\lim _{x \rightarrow B} \gamma x^{\alpha} \in \mathrm{cl} I$, which contradicts $I \neq(0,+\infty)$. Similarly, if $A=0$, then (2.3) implies $+\infty=\lim _{x \rightarrow A^{+}} \gamma x^{\alpha} \in \operatorname{cl} I$, which contradicts $I \neq(0,+\infty)$.

Applying condition (2.3), we get $0<A \leq \gamma B^{\alpha}$ and $\gamma A^{\alpha} \leq B<+\infty$. Hence $\gamma A^{\alpha} \leq B \leq$ $\gamma^{-\frac{1}{\alpha}} A^{\frac{1}{\alpha}}$.

(i) If $\alpha<-1$, then $\gamma^{\frac{1}{1-\alpha}} \leq A \leq B \leq \gamma^{\frac{1}{1-\alpha}}$.

(ii) If $\alpha=-1$, then $\frac{\gamma}{A} \leq B \leq \frac{\gamma}{A}$.

(iii) If $\alpha \in(-1,0)$, then $A \leq \gamma^{\frac{1}{1-\alpha}} \leq B$.

Assume now that $\alpha \geq 0$.

Then (2.3) yields $A \leq \gamma A^{\alpha}$ provided that $A>0$ and $\gamma B^{\alpha} \leq B$ provided that $B<+\infty$.

(iv) If $\alpha \in[0,1)$, then $A \leq \gamma^{\frac{1}{1-\alpha}}$ if $A>0$ and $\gamma^{\frac{1}{1-\alpha}} \leq B$ if $B<+\infty$.

(v) If $\alpha=1<\gamma$, then $B=+\infty$ and no restriction on $A$.

(vi) If $\alpha=1=\gamma$, then no restriction on $A$ and $B$. 
(vii) If $\alpha=1>\gamma$, then $A=0$ and no restriction on $B$.

(viii) If $\alpha>1$, then $A \geq \gamma^{\frac{1}{1-\alpha}}$ if $A>0$ and $B \leq \gamma^{\frac{1}{1-\alpha}}$ if $B<+\infty$.

As a consequence of Corollary 2.2, we have the following direct construction of all continuous solutions $f:(0,+\infty) \rightarrow(0,+\infty)$ of equation (2.1) ( $c f$. [13, Theorem 15.15]).

Corollary 2.3 Let I be a suitably chosen interval from Corollary 2.2 for a given $\alpha \in \mathbb{R}$, and let $f_{0}: I \rightarrow I$ be a function given by $f_{0}(x)=\gamma x^{\alpha}$. Then every extension of $f_{0}$ to a continuous function $f:(0,+\infty) \rightarrow(0,+\infty)$ satisfying $(2.5)$ is a solution of equation (2.1).

\subsection{The case $\alpha=\mathbf{0} \neq \boldsymbol{\beta}$}

If $\alpha=0 \neq \beta$, then equation (1.1) reduces to the equation

$$
f^{2}(x)=\gamma x^{\beta}
$$

Charles Babbage was probably the first who looked for solutions of equation (2.6) in the case where $\beta=\gamma=1$ (see [14]). For the case where $\gamma=-\beta=1$, see [15-17]; $c f$. also [18]. Equation (2.6) is a particular case of the equation of iterative roots (see [12, 13, 19-21]). According to known results on the equation of iterative roots, we can formulate a theorem on continuous solutions $f:(0,+\infty) \rightarrow(0,+\infty)$ of equation (2.6).

Denote by $I(x, y)$ the closed interval $[\min \{x, y\}, \max \{x, y\}]$ with $x, y \in(0,+\infty)$.

\section{Theorem 2.4}

(i) Assume $\beta<0$. Then equation (2.6) has no continuous solution $f:(0,+\infty) \rightarrow(0,+\infty)$.

(ii) Assume $\beta=\gamma=1$.

(ii $\left.i_{1}\right)$ Then the formula $f(x)=x$ defines the unique continuous solution $f:(0,+\infty) \rightarrow$ $(0,+\infty)$ of equation (2.6).

(ii ${ }_{2}$ ) If $f:(0,+\infty) \rightarrow(0,+\infty)$ is a continuous and decreasing solution of equation (2.6), then there exists $x_{0} \in(0,+\infty)$ such that $f\left(x_{0}\right)=x_{0}$ and $f$ maps $\left(0, x_{0}\right]$ bijectively onto $\left[x_{0},+\infty\right)$. Conversely, if $x_{0} \in(0,+\infty)$, then every decreasing bijection $f_{0}:\left(0, x_{0}\right] \rightarrow\left[x_{0},+\infty\right)$ such that $f\left(x_{0}\right)=x_{0}$ can be uniquely extended to a continuous and decreasing solution $f:(0,+\infty) \rightarrow(0,+\infty)$ of equation (2.6).

(iii) Assume $\beta=1 \neq \gamma$.

(iii $\left.{ }_{1}\right)$ Let $x_{0} \in(0,+\infty)$. If $:(0,+\infty) \rightarrow(0,+\infty)$ is a continuous and increasing solution of equation (2.6), then $f$ maps $I\left(x_{0}, f\left(x_{0}\right)\right)$ bijectively onto $I\left(f\left(x_{0}\right), \gamma x_{0}\right)$. Conversely, if $x_{1} \in \operatorname{Int} I\left(x_{0}, \gamma x_{0}\right)$, then every increasing bijection $f_{0}: I\left(x_{0}, x_{1}\right) \rightarrow I\left(x_{1}, \gamma x_{0}\right)$ can be uniquely extended to a continuous and increasing solution $f:(0,+\infty) \rightarrow$ $(0,+\infty)$ of equation (2.6).

(iii $\left._{2}\right)$ Equation (2.6) has no continuous and decreasing solution from $f:(0,+\infty) \rightarrow$ $(0,+\infty)$.

(iv) Assume $0<\beta \neq 1$.

$\left(\mathrm{iv}_{1}\right)$ Let $x_{0} \in\left(0, \gamma^{\frac{1}{1-\beta}}\right)$ and let $y_{0} \in\left(\gamma^{\frac{1}{1-\beta}},+\infty\right)$. Iff $:(0,+\infty) \rightarrow(0,+\infty)$ is a continuous and increasing solution of equation (2.6), then $f$ maps $I\left(x_{0}, f\left(x_{0}\right)\right) \cup I\left(y_{0}, f\left(y_{0}\right)\right)$ bijectively onto $I\left(f\left(x_{0}\right), \gamma x_{0}^{\beta}\right) \cup I\left(f\left(y_{0}\right), \gamma y_{0}^{\beta}\right)$. Conversely, if $x_{1} \in \operatorname{Int} I\left(x_{0}, \gamma x_{0}^{\beta}\right)$ 
and $y_{1} \in \operatorname{Int} I\left(y_{0}, \gamma y_{0}^{\beta}\right)$, then every increasing bijection $f_{0}: I\left(x_{0}, x_{1}\right) \cup I\left(y_{0}, y_{1}\right) \rightarrow$ $I\left(x_{1}, \gamma x_{0}^{\beta}\right) \cup I\left(y_{1}, \gamma y_{0}^{\beta}\right)$ can be uniquely extended to a continuous and increasing solution $f:(0,+\infty) \rightarrow(0,+\infty)$ of equation (2.6).

$\left(\mathrm{iv}_{2}\right)$ If $:(0,+\infty) \rightarrow(0,+\infty)$ is a continuous and decreasing solution of equation (2.6), then $f$ maps $\left(0, \gamma^{\frac{1}{1-\beta}}\right]$ bijectively onto $\left[\gamma^{\frac{1}{1-\beta}},+\infty\right)$ and $\gamma[f(x)]^{\beta}=f\left(\gamma x^{\beta}\right)$ for all $x \in(0,+\infty)$. Conversely, every decreasing bijection $f_{0}:\left(0, \gamma^{\frac{1}{1-\beta}}\right] \rightarrow\left[\gamma^{\frac{1}{1-\beta}},+\infty\right)$ such that $\gamma\left[f_{0}(x)\right]^{\beta}=f_{0}\left(\gamma x^{\beta}\right)$ for all $x \in\left(0, \gamma^{\frac{1}{1-\beta}}\right]$ can be uniquely extended to a continuous and decreasing solution $f:(0,+\infty) \rightarrow(0,+\infty)$ of equation (2.6).

Proof All the assertions can be derived from [13, Chapter XV]) as it has been noticed earlier. However, most of the assertions have evident proofs, so we present them for the convenience of the reader.

(i) Suppose that, contrary to our claim, equation (2.6) has a continuous solution $f:(0,+\infty) \rightarrow(0,+\infty)$. Then the second iterate $f^{2}$ of $f$ is strictly increasing. Now by (2.6) we conclude that $\beta>0$, a contradiction.

$\left(\mathrm{ii}_{1}\right)$ It is clear that the identity function on $(0,+\infty)$ satisfies $(2.6)$. Suppose the contrary, and let $f:(0,+\infty) \rightarrow(0,+\infty)$ be another increasing solution of equation (2.6). Then there exists $x \in(0,+\infty)$ such that $f(x) \neq x$. By the monotonicity of $f$, we conclude that $f^{2}(x) \neq x$, which contradicts (2.6).

$\left(\mathrm{ii}_{2}\right)$ The first assertion is clear. To prove the second one, fix a decreasing bijection $f_{0}:\left(0, x_{0}\right] \rightarrow\left[x_{0},+\infty\right)$ such that $f\left(x_{0}\right)=x_{0}$ and extend it to a function $f:(0,+\infty) \rightarrow$ $(0,+\infty)$ putting $f(x)=f_{0}^{-1}(x)$ for all $x \in\left(x_{0},+\infty\right)$. It is easy to see that $f$ is a decreasing bijection satisfying (2.6).

(iii $\left.{ }_{1}\right)$ The first assertion is evident. The second one can be deduced from [13, Lemma 15.6].

(iii $\left.{ }_{2}\right)$ Suppose, to derive a contradiction, that $f:(0,+\infty) \rightarrow(0,+\infty)$ is a continuous and decreasing solution of equation (2.6). Then there exists $x \in(0,+\infty)$ such that $f(x)=x$. Hence $f^{2}(x)=x \neq \gamma x$, a contradiction.

$\left(\mathrm{iv}_{1}\right)$ The first assertion is easy to verify. The second one can be inferred from [13, Theorem 15.7].

$\left(\mathrm{iv}_{2}\right)$ Let $f$ be a continuous and decreasing solution of equation (2.6). Then there exists $x_{0} \in(0,+\infty)$ such that $f\left(x_{0}\right)=x_{0}$. Hence by (2.6) we get $x_{0}=f^{2}\left(x_{0}\right)=\gamma x_{0}^{\beta}$, and thus $x_{0}=$ $\gamma^{\frac{1}{1-\beta}}$. Consequently, $f$ maps $\left(0, \gamma^{\frac{1}{1-\beta}}\right]$ bijectively onto $\left[\gamma^{\frac{1}{1-\beta}},+\infty\right)$. Moreover, (2.6) yields $f\left(\gamma x^{\beta}\right)=f^{3}(x)=\gamma[f(x)]^{\beta}$ for all $x \in(0,+\infty)$. To prove the second part of the assertion, fix a decreasing bijection $f_{0}:\left(0, \gamma^{\frac{1}{1-\beta}}\right] \rightarrow\left[\gamma^{\frac{1}{1-\beta}},+\infty\right)$ such that $\gamma\left[f_{0}(x)\right]^{\beta}=f_{0}\left(\gamma x^{\beta}\right)$ for all $x \in\left(0, \gamma^{\frac{1}{1-\beta}}\right]$ and extend it to a function $f:(0,+\infty) \rightarrow(0,+\infty)$ putting $f(x)=f_{0}^{-1}\left(\gamma x^{\beta}\right)$ for all $x \in\left(\gamma^{\frac{1}{1-\beta}},+\infty\right)$. It is easy to calculate that $f$ is a decreasing bijection satisfying (2.6).

\subsection{The case $\alpha \neq 0 \neq \beta$}

In this case an explicit description of all continuous solutions $f:(0,+\infty) \rightarrow(0,+\infty)$ of equation (1.1) is much more involved than in both the previous cases. We begin with an observation which allows us to rewrite equation (1.1) in an equivalent form. 
Lemma 2.5 If $f:(0,+\infty) \rightarrow(0,+\infty)$ is a solution of equation (1.1), then the function $g: \mathbb{R} \rightarrow \mathbb{R}$ given by $g(x)=\log f\left(e^{x}\right)$ satisfies

$$
g^{2}(x)=\log \gamma+\alpha g(x)+\beta x .
$$

Conversely, if $g: \mathbb{R} \rightarrow \mathbb{R}$ is a solution of equation (2.7), then the function $f:(0,+\infty) \rightarrow$ $(0,+\infty)$ given by $f(x)=e^{g(\log x)}$ satisfies $(1.1)$.

Equation (2.7) is a special case of the polynomial-like iterative inhomogeneous equation

$$
\sum_{n=0}^{N} a_{n} f^{n}(x)=b,
$$

where all $a_{n}$ 's and $b$ are real numbers and $f$ is an unknown self-mapping; here $f^{n}$ denotes the $n$th iterate of $f$. For the theory of equation (2.8) and its generalizations, we refer the readers to [22-30]. The problem of finding all continuous solutions of equation (2.8) seems to be very difficult. It is completely solved in [31] for $N=2$, but it is still open even in the case where $N=3$ (see [32]). It turns out that the nature of continuous solutions of equation (2.8) depends on the behavior of complex roots $r_{1}, \ldots, r_{N}$ of its characteristic equation $\sum_{n=0}^{N} \alpha_{n} r^{n}=0$. This characteristic equation is obtained by putting $f(x)=r x$ into (2.8) with $b=0$; in this way we can determine all linear solutions of the homogeneous counterpart of equation (2.8), and then all affine solutions of equation (2.8). Therefore, to formulate our result on continuous solutions $f:(0,+\infty) \rightarrow(0,+\infty)$ of equation (1.1), denote by $r_{1}$ and $r_{2}$ the complex roots of the equation

$$
r^{2}-\alpha r-\beta=0
$$

By our assumption, we have $r_{1}+r_{2}=\alpha \neq 0$ and $r_{1} r_{2}=-\beta \neq 0$.

Combining Lemma 2.5 with the results from [31], we get the following theorem.

\section{Theorem 2.6}

(i) Assume $r_{1}, r_{2} \in \mathbb{C} \backslash \mathbb{R}$. Then equation (1.1) has no continuous solution $f:(0,+\infty) \rightarrow$ $(0,+\infty)$.

(ii) Assume $r_{1}, r_{2} \in \mathbb{R} \backslash\{1\}$. Let $\xi=\gamma^{\frac{1}{\left(r_{1}-1\right)\left(r_{2}-1\right)}}$ and $f_{1}, f_{2}:(0,+\infty) \rightarrow(0,+\infty)$ be defined by

$$
f_{1}(x)=\gamma^{-\frac{1}{r_{2}-1}} x^{r_{1}}, \quad f_{2}(x)=\gamma^{-\frac{1}{r_{1}-1}} x^{r_{2}} .
$$

(ii 1 ) Assume either $1<r_{1}<r_{2}$ or $0<r_{1}<r_{2}<1$. Let $0<x_{0}<\xi<y_{0}$. If $f:(0,+\infty) \rightarrow$ $(0,+\infty)$ is a continuous solution of equation (1.1), then $f(\xi)=\xi, f_{0}=$ $\left.f\right|_{I\left(x_{0}, f\left(x_{0}\right)\right) \cup I\left(y_{0}, f\left(y_{0}\right)\right)}$ is continuous,

$$
\begin{array}{ll}
f_{0}\left(x_{0}\right) \in I\left(f_{1}\left(x_{0}\right), f_{2}\left(x_{0}\right)\right), & f_{0}^{2}\left(x_{0}\right)=\gamma\left[f_{0}\left(x_{0}\right)\right]^{\alpha} x_{0}^{\beta}, \\
f_{0}\left(y_{0}\right) \in I\left(f_{1}\left(y_{0}\right), f_{2}\left(y_{0}\right)\right), & f_{0}^{2}\left(y_{0}\right)=\gamma\left[f_{0}\left(y_{0}\right)\right]^{\alpha} y_{0}^{\beta}
\end{array}
$$


and

$$
\left(\frac{y}{x}\right)^{r_{1}} \leq \frac{f_{0}(y)}{f_{0}(x)} \leq\left(\frac{y}{x}\right)^{r_{2}}
$$

for all $x, y \in I\left(x_{0}, f\left(x_{0}\right)\right) \cup I\left(y_{0}, f\left(y_{0}\right)\right)$. Conversely, every continuous function $f_{0}: I\left(x_{0}, f_{0}\left(x_{0}\right)\right) \cup I\left(y_{0}, f_{0}\left(y_{0}\right)\right) \rightarrow \mathbb{R}$ such that (2.9) and (2.10) are satisfied and (2.11) holds for all $x, y \in I\left(x_{0}, f_{0}\left(x_{0}\right)\right) \cup I\left(y_{0}, f_{0}\left(y_{0}\right)\right)$ can be uniquely extended to a continuous solution $f:(0,+\infty) \rightarrow(0,+\infty)$ of equation (1.1).

$\left(\mathrm{ii}_{2}\right)$ Assume $0<r_{1}<1<r_{2}$. Let $x_{0}>0$. If $f:(0,+\infty) \rightarrow(0,+\infty)$ is a continuous solution of equation (1.1), then either $\left.f\right|_{(0, \xi]} \in\left\{\left.f_{1}\right|_{(0, \xi]},\left.f_{2}\right|_{(0, \xi]}\right\}$ and $\left.f\right|_{(\xi,+\infty)} \in$ $\left\{\left.f_{1}\right|_{(\xi,+\infty)},\left.f_{2}\right|_{(\xi,+\infty)}\right\}$ or $f_{0}=\left.f\right|_{I\left(x_{0}, f\left(x_{0}\right)\right)}$ is continuous,

$$
f_{0}\left(x_{0}\right) \notin I\left(f_{1}\left(x_{0}\right), f_{2}\left(x_{0}\right)\right), \quad f_{0}^{2}\left(x_{0}\right)=\gamma\left[f_{0}\left(x_{0}\right)\right]^{\alpha} x_{0}^{\beta}
$$

and (2.11) holds for all $x, y \in I\left(x_{0}, f\left(x_{0}\right)\right)$. Conversely, every continuous function $f_{0}: I\left(x_{0}, f_{0}\left(x_{0}\right)\right) \rightarrow \mathbb{R}$ such that (2.12) is satisfied and (2.11) holds for all $x, y \in$ $I\left(x_{0}, f_{0}\left(x_{0}\right)\right)$ can be uniquely extended to a continuous solution $f:(0,+\infty) \rightarrow$ $(0,+\infty)$ of equation (1.1).

$\left(\mathrm{ii}_{3}\right)$ Assume either $r_{1}<r_{2}<-1$ or $-1<r_{1}<r_{2}<0$. Let $x_{0}>\xi$. Iff: $(0,+\infty) \rightarrow(0,+\infty)$ is a continuous solution of equation (1.1), then $f(\xi)=\xi$ and $f_{0}=\left.f\right|_{I\left(x_{0}, f^{2}\left(x_{0}\right)\right)}$ is continuous,

$$
f_{0}\left(x_{0}\right) \in I\left(f_{1}\left(x_{0}\right), f_{2}\left(x_{0}\right)\right), \quad f_{0}^{3}\left(x_{0}\right)=\gamma\left[f_{0}^{2}\left(x_{0}\right)\right]^{\alpha} x_{0}^{\beta},
$$

and (2.11) holds for all $x, y \in I\left(x_{0}, f^{2}\left(x_{0}\right)\right)$. Conversely, every continuous function $f_{0}: I\left(x_{0}, f_{0}^{2}\left(x_{0}\right)\right) \rightarrow \mathbb{R}$ such that (2.13) is satisfied and (2.11) holds for all $x, y \in$ $I\left(x_{0}, f_{0}^{2}\left(x_{0}\right)\right)$ can be uniquely extended to a continuous solution $f:(0,+\infty) \rightarrow$ $(0,+\infty)$ of equation (1.1).

(ii $4_{4}$ ) Assume either $r_{1}<r_{2}=-1$ or $-1=r_{2}<r_{1}<0$. Then every continuous solution $f:(0,+\infty) \rightarrow(0,+\infty)$ of equation (1.1) is of the form

$$
f(x)= \begin{cases}a^{r_{1}+1} \xi^{1-r_{1}} x^{r_{1}} & \text { for } x \in\left(0, a^{-1} \xi\right], \\ \xi^{2} x^{-1} & \text { for } x \in\left(a^{-1} \xi, a \xi\right), \\ a^{-r_{1}-1} \xi^{1-r_{1}} x^{r_{1}} & \text { for } x \in[a \xi,+\infty)\end{cases}
$$

with some $a \in[1,+\infty]$.

(ii $\left.{ }_{5}\right)$ Assume either $r_{1}<0<r_{2}$ or $r_{1}<-1<r_{2}<0$. Then $f_{1}$ and $f_{2}$ are the only continuous solutions from $(0,+\infty)$ to $(0,+\infty)$ of equation (1.1).

(iii) Assume $r_{2}=1 \neq r_{1}$. Let $f_{3}:(0,+\infty) \rightarrow(0,+\infty)$ be defined by

$$
f_{3}(x)=\gamma^{-\frac{1}{r_{1}-1}} x
$$

(iii 1 ) Assume $r_{1}>1 \neq \gamma$. If $f:(0,+\infty) \rightarrow(0,+\infty)$ is a continuous solution of equation (1.1), then $f_{0}=\left.f\right|_{I\left(x_{0}, f\left(x_{0}\right)\right)}$ is continuous,

$$
f_{0}\left(x_{0}\right) \leq f_{3}\left(x_{0}\right), \quad f_{0}^{2}\left(x_{0}\right)=\gamma\left[f_{0}\left(x_{0}\right)\right]^{\alpha} x_{0}^{\beta}
$$


and

$$
\frac{y}{x} \leq \frac{f_{0}(y)}{f_{0}(x)} \leq\left(\frac{y}{x}\right)^{r_{1}}
$$

for all $x, y \in I\left(x_{0}, f\left(x_{0}\right)\right)$. Conversely, every continuous function $f_{0}: I\left(x_{0}, f_{0}\left(x_{0}\right)\right) \rightarrow$ $\mathbb{R}$ such that (2.14) is satisfied and (2.15) holds for all $x, y \in I\left(x_{0}, f_{0}\left(x_{0}\right)\right)$ can be uniquely extended to a continuous solution $f:(0,+\infty) \rightarrow(0,+\infty)$ of equation (1.1).

(iii $)_{2}$ Assume $0<r_{1}<1 \neq \gamma$. Iff: $(0,+\infty) \rightarrow(0,+\infty)$ is a continuous solution of equation (1.1), then $f_{0}=\left.f\right|_{I\left(x_{0}, f\left(x_{0}\right)\right)}$ is continuous,

$$
f_{0}\left(x_{0}\right) \geq f_{3}\left(x_{0}\right), \quad f_{0}^{2}\left(x_{0}\right)=\gamma\left[f_{0}\left(x_{0}\right)\right]^{\alpha} x_{0}^{\beta}
$$

and

$$
\left(\frac{y}{x}\right)^{r_{1}} \leq \frac{f_{0}(y)}{f_{0}(x)} \leq \frac{y}{x}
$$

for all $x, y \in I\left(x_{0}, f\left(x_{0}\right)\right)$. Conversely, every continuous function $f_{0}: I\left(x_{0}, f_{0}\left(x_{0}\right)\right) \rightarrow$ $\mathbb{R}$ such that (2.16) is satisfied and (2.17) holds for all $x, y \in I\left(x_{0}, f_{0}\left(x_{0}\right)\right)$ can be uniquely extended to a continuous solution $f:(0,+\infty) \rightarrow(0,+\infty)$ of equation (1.1).

(iii $\left.{ }_{3}\right)$ Assume $r_{1}>0$ and $\gamma=1$. Then every continuous solution $f:(0,+\infty) \rightarrow(0,+\infty)$ of equation (1.1) is of the form

$$
f(x)= \begin{cases}a^{1-r_{1}} x^{r_{1}} & \text { for } x \in(0, a] \\ x & \text { for } x \in(a, b), \\ b^{1-r_{1}} x^{r_{1}} & \text { for } x \in[b,+\infty)\end{cases}
$$

with some $0 \leq a \leq b \leq+\infty$.

(iii $\left.{ }_{4}\right)$ Assume $r_{1}<0$ and $\gamma \neq 1$. Then $f_{3}$ is the unique continuous solution from $(0,+\infty)$ to $(0,+\infty)$ of equation (1.1).

(iii $\left.{ }_{5}\right)$ Assume $r_{1}<0$ and $\gamma=1$. Then $f_{3}$ is a continuous solution of equation (1.1) and every other continuous solution $f:(0,+\infty) \rightarrow(0,+\infty)$ of equation (1.1) is of the form

$$
f(x)=a x^{r_{1}}
$$

with some $a \in(0,+\infty)$.

(iv) Assume $r_{1}=r_{2}$.

(iv $)$ Assume $r_{1} \neq 1$. Then the formula

$$
f(x)=\gamma^{-\frac{1}{r_{1}-1}} x^{r_{1}}
$$

defines the unique continuous solution $f:(0,+\infty) \rightarrow(0,+\infty)$ of equation (1.1). 
$\left(\mathrm{iv}_{2}\right)$ Assume $r_{1}=1 \neq \gamma$. Then equation (1.1) has no continuous solution $f:(0,+\infty) \rightarrow$ $(0,+\infty)$.

$\left(\mathrm{iv}_{3}\right)$ Assume $r_{1}=\gamma=1$. Then every continuous solution $f:(0,+\infty) \rightarrow(0,+\infty)$ of equation (1.1) is of the form

$$
f(x)=a x
$$

with some $a \in(0,+\infty)$.

\section{Competing interests}

The author declares that he has no competing interests.

\section{Author's contributions}

The author wrote the whole article, read and approved the final manuscript.

\section{Acknowledgements}

This research was supported by Silesian University Mathematics Department (Iterative Functional Equations and Real Analysis program).

Received: 9 August 2014 Accepted: 14 October 2014 Published: 22 Oct 2014

\section{References}

1. Brillouët-Belluot, N: Problem posed during The Forty-ninth International Symposium on Functional Equations. Aequ. Math. 84, 312 (2012)

2. Brodsky, YS, Slipenko, AK: Functional Equations. Vishcha Shkola, Kiev (1983)

3. Boros, Z: Talk given during The Fiftieth International Symposium on Functional Equations. Aequ. Math. 86, 293 (2013)

4. Sablik, M: Talk given during The Fourteenth Debrecen-Katowice Winter Seminar on Functional Equations and Inequalities. Hajdúszoboszló, Hungary, January 29 - February 1 (2014)

5. Jarczyk, W, Morawiec, J: Note on an equation occurring in a problem of Nicole Brillouët-Belluot. Aequ. Math. 84, 227-233 (2012)

6. Morawiec, J: On a problem of Nicole Brillouët-Belluot. Aequ. Math. 84, 219-225 (2012)

7. Morawiec, J: Around a problem of Nicole Brillouët-Belluot. Aequ. Math. 88, 175-181 (2014)

8. Morawiec, J: Around a problem of Nicole Brillouët-Belluot, II. Aequ. Math. (2014). doi:10.1007/s00010-013-0249-z

9. Chen, L, Shi, Y: The real solutions of functional equation $f^{[m]}=1 / f$. J. Math. Res. Expo. 28, 323-330 (2008)

10. Ng, CT, Zhang, W: When does an iterate equal a power? Publ. Math. (Debr.) 67, 79-91 (2005)

11. Shi, Y, Chen, L: All the meromorphic solutions of the functional equation $f^{[m]}=1 / f$. J. Nat. Sci. Nanjing Norm. Univ. 2 , 25-27 (2007)

12. Baron, K, Jarczyk, W: Recent results on functional equations in a single variable, perspectives and open problem. Aequ. Math. 61, 1-48 (2001)

13. Kuczma, M: Functional Equations in a Single Variable. Monografie Matematyczne, vol. 46. PWN, Warsaw (1968)

14. Babbage, C: Essay towards the calculus of functions. Philos. Trans. R. Soc. Lond. 105, 389-424 (1815)

15. Anschuetz, R, Scherwood, H: When is a function's inverse equal to its reciprocal? Coll. Math. J. 27, 388-393 (1997)

16. Cheng, R, Dasgupta, A, Ebanks, BR, Kinch, LF, Larso, LM, McFadden, RB: When does $f^{-1}=1 / f$ ? Am. Math. Mon. 105, 704-716 (1998)

17. Euler, R, Foran, J: On functions whose inverse is their reciprocal. Math. Mag. 54, 185-189 (1981)

18. Massera, JL, Petracca, A: Sobre la ecuación funcional $f(f(x))=1 / x$. Rev. Unión Mat. Argent. 11, 206-211 (1946)

19. Kuczma, M, Choczewski, B, Ger, R: Iterative Functional Equations. Encyclopedia of Mathematics and Its Applications, vol. 32. Cambridge University Press, Cambridge (1990)

20. Targonski, G: Topics in Iteration Theory. Vandenhoeck \& Ruprecht, Göttingen (1981)

21. Targonski, G: Progress of iteration theory since 1981. Aequ. Math. 50, 50-72 (1995)

22. Chen, J, Zhang, W: Leading coefficient problem for polynomial-like iterative equations. J. Math. Anal. Appl. 349 413-419 (2009)

23. Jarczyk, W: A Recurrent Method of Solving Iterative Functional Equations. Prace Naukowe Uniwersytetu Śląskiego W Katowicach 1206, Uniwersytet Śląski, Katowice (1991)

24. Matkowski, J, Zhang, Z: Characteristic analysis for a polynomial-like iterative equation. Chin. Sci. Bull. 43, 192-196 (1998)

25. Trif, T: Convex solutions to polynomial-like iterative equations on open intervals. Aequ. Math. 79, 315-325 (2010)

26. Xu, B, Zhang, W: Construction of continuous solutions and stability for the polynomial like iterative equation. J. Math. Anal. Appl. 325, 1160-1170 (2007)

27. Yang, D, Zhang, W: Characteristic solutions of polynomial-like iterative equations. Aequ. Math. 67, 80-105 (2004)

28. Tabor, J, Tabor, J: On a linear iterative equation. Results Math. 27, 412-421 (1995)

29. Zhang, W, Zhang, W: On continuous solutions of $n$-th order polynomial-like iterative equations. Publ. Math. (Debr.) 76, 117-134 (2010)

30. Zhang, $\mathrm{P}$ Gong, X: Continuous solutions of 3-order iterative equation of linear dependence. Manuscript

31. Nabeya, S: On the functional equation $f(p+q x+r f(x))=a+b x+c f(x)$. Aequ. Math. 11, 199-211 (1974)

32. Matkowski, J: Remark done during The Twenty-sixth International Symposium on Functional Equations. Aequ. Math. 37, 119 (1989) 
10.1186/1687-1847-2014-27

Cite this article as: Morawiec: On a functional equation involving iterates and powers. Advances in Difference Equations 2014, 2014:271

Submit your manuscript to a SpringerOpen ${ }^{\circ}$ journal and benefit from:

- Convenient online submission

- Rigorous peer review

- Immediate publication on acceptance

- Open access: articles freely available online

- High visibility within the field

- Retaining the copyright to your article

Submit your next manuscript at $\gg$ springeropen.com 